\title{
EFICIENCIA DE Eisenia foetida, Eichornia crassipes E HIPOCLORITO DE CALCIO EN LA DEPURACIÓN DE AGUAS RESIDUALES DOMÉSTICAS EN MOQUEGUA, PERÚ
}

\author{
EFFICIENCY OF Eisenia foetida, Eichornia crassipes AND CALCIUM \\ HYPOCHLORITE IN THE DEPURATION OF DOMESTIC WASTEWATER IN \\ MOQUEGUA, PERU
}

\author{
Deybi Karin Cáceres Poma ${ }^{1}$, Gisela Milagros Calisaya Vera² y Edgar Bedoya-Justo ${ }^{3}$
}

\begin{abstract}
Resumen
Se determinó la eficiencia de tres sistemas de depuración de aguas residuales domésticas con Eisenia foetida, Eichornia crassipes e hipoclorito de calcio en Moquegua. Se tomó una muestra representativa de aguas residuales domésticas (afluente) y se analizaron parámetros físicos (temperatura y STS), químicos $\left(\mathrm{pH}\right.$ y $\mathrm{DBO}_{5}$ ) y microbiológicos (coliformes termotolerantes), para determinar su estado inicial. Este afluente -previo proceso de pre-sedimentación-fue tratado en tres sistemas de depuración: el primero con E. foetida, el segundo con E. foetida + E. crassipes, y el tercero con E. foetida + E. crassipes $+\mathrm{Ca}(\mathrm{ClO})_{2}$. E. foetida, a su vez, fue evaluada en función de distintas tasas de riego: $\mathrm{A}_{1}\left(0.5\left[\mathrm{~m}^{3} / \mathrm{día}\right] / \mathrm{m}^{2}\right), \mathrm{A}_{2}\left(1\left[\mathrm{~m}^{3} / \mathrm{día}\right] / \mathrm{m}^{2}\right)$ y $\mathrm{A}_{0}$ (grupo control: sin tratamiento); E. crassipes, en función del tiempo de retención: $\mathrm{B}_{1}$ (7 días), $\mathrm{B}_{2}$ (14 días) y $\mathrm{B}_{0}$ (grupo control: sin tratamiento); y $\mathrm{Ca}(\mathrm{ClO})_{2}$ en función de $\mathrm{C}_{1}$ (aplicación de hipoclorito de calcio) y $\mathrm{C}_{0}$ (grupo control: sin tratamiento). Los efluentes fueron analizados considerando los mismos parámetros que en el afluente, cada uno con dos repeticiones, aplicándose el análisis de varianza (ANOVA) como prueba estadística y la prueba de Tukey. Los resultados obtenidos mostraron que existen diferencias significativas entre los sistemas de depuración propuestos, determinándose como el más eficiente al conformado por E. foetida + E. crassipes, con un descenso medio de $5^{\circ} \mathrm{C}, 94.48 \%$ de STS, $98.41 \%$ de $\mathrm{DBO}_{5}, 100.00 \%$ de coliformes termotolerantes, y un pH final de 7.51 .
\end{abstract}

Palabras clave: tratamiento de agua residual, Eisenia foetida, Eichhornia crassipes, hipoclorito de calcio.

\begin{abstract}
The efficiency of three domestic wastewater treatment systems with Eisenia foetida, Eichornia crassipes and calcium hypochlorite in Moquegua was determined. A representative sample of domestic wastewater (tributary) was taken and physical (temperature and STS), chemical $\left(\mathrm{pH}\right.$ and $\mathrm{BOD}_{5}$ ) and microbiological (thermotolerant coliforms) parameters were analyzed to determine its initial state. This tributary -previous pre-sedimentation process- was treated in three depuration systems: the first with $E$. foetida, the second with $E$. foetida $+E$. crassipes, and the third with E. foetida + E. crassipes $+\mathrm{Ca}(\mathrm{ClO})_{2}$. E. foetida, in turn, was evaluated in function of different irrigation rates: $\mathrm{A}_{1}\left(0.5\left[\mathrm{~m}^{3} / \mathrm{day}\right] / \mathrm{m}^{2}\right), \mathrm{A}_{2}\left(1\left[\mathrm{~m}^{3} / \mathrm{day}\right] / \mathrm{m}^{2}\right)$ and $\mathrm{A}_{0}$ (control group: no treatment); E. crassipes was evaluated in function of retention time: $\mathrm{B}_{1}$ ( 7 days), $\mathrm{B}_{2}$ (14 days) and $\mathrm{B}_{0}$ (control group: no treatment); and $\mathrm{Ca}(\mathrm{ClO})_{2}$ was evaluated in function of $\mathrm{C}_{1}$ (application of calcium hypochlorite) and $\mathrm{C}_{0}$ (control group: no treatment). The effluents were analyzed considering the same parameters as in the tributary, each one with two repetitions, applying the analysis of variance (ANOVA) as a statistical test and the Tukey test. The obtained results showed that there are significant differences between the proposed depuration systems, being determined as the most efficient system to the conformed by E. foetida $+E$. crassipes, with an average decrease of $5^{\circ} \mathrm{C}$, $94.48 \%$ of total suspended solids, $98.41 \%$ of $\mathrm{BOD}_{5}, 100.00 \%$ of thermotolerant coliforms, and a final $\mathrm{pH}$ of 7.51 .
\end{abstract}

Key words: wastewater treatment, Eisenia foetida, Eichhornia crassipes, calcium hypochlorite.

\section{Introducción}

El Decreto Supremo No 001-2010-AG (MINAGRI, 2010), que aprueba el Reglamento de la Ley de Recursos Hídricos (Ley N 29338), establece que las aguas residuales domésticas son "aquellas de origen residencial, comercial e institucional que contienen desechos fisiológicos y otros provenientes de la actividad humana".
En Perú, de acuerdo al Organismo de Evaluación y Fiscalización Ambiental, las Entidades Prestadoras de Servicios (EPS) de Saneamiento solo dan cobertura al $69.65 \%$ de la población urbana y, a su vez, solo el $32 \%$ de las aguas residuales descargadas al sistema de alcantarillado recibe tratamiento previo (OEFA, 2014). Además, en aquellas plantas de tratamiento de aguas residuales (PTAR), cuya infraestructura es insuficiente, 
se presentan problemas de sobrecarga, lo cual origina que los efluentes no se traten adecuadamente. Por otro lado, la población no cubierta vierte sus aguas residuales sin tratamiento a los cuerpos de agua natural, quebradas y terrenos baldíos, o las emplean en el riego de cultivos, representando un riesgo para la salud y el ambiente (OEFA, 2014).

Sin embargo, las aguas residuales domésticas precisan de un tratamiento adecuado antes de su disposición final o reúso (MINAM, 2009). Por ello, ante esta problemática, es que se propuso la presente investigación, realizada en el año 2017, la cual tuvo como objetivo evaluar tres sistemas de depuración para determinar su eficiencia en el tratamiento de aguas residuales domésticas. Éstos incluyeron la depuración con lombrices (Eiseniafoetida), jacinto de agua (Eichhornia crassipes) y cloración.

La lombriz roja californiana (Eisenia foetida Savigny 1826) se caracteriza por su rusticidad, flexibilidad a los factores ambientales (humedad, temperatura y $\mathrm{pH}$ ), y capacidad de reproducción y apiñamiento. Este anélido fotofóbico se alimenta de todo tipo de desechos orgánicos de origen animal o vegetal con $70 \%$ de contenido de humedad (Rodríguez, 2005) y es capaz de producir deyecciones ricas en flora bacteriana, consideradas como un excelente abono orgánico (García \& Solano, 2005). Los sistemas de tratamiento de aguas residuales con lombricultura son sistemas biológicos de flujo vertical basados en la aplicación de lombrices (Vizcaíno \& Fuentes, 2016), los cuales, debido a sus características físicas o estructurales, son altamente eficientes en la remoción de materia orgánica y organismos patógenos (Fernández, 2011, citado por Ramón et al., 2015). El agua residual es regada sobre el biofiltro compuesto por estratos de diversos materiales, en cuya capa superior se encuentra el material orgánico con un elevado número de microorganismos y lombrices. Este afluente percola a través de los distintos estratos del medio filtrante, quedando retenida en las capas superiores la materia orgánica contenida en el agua residual, que luego será consumida por las lombrices y organismos asociados (Fernández, 2011, citado por Ramón et al., 2015).

El jacinto de agua (Eichhornia crassipes (Martius) Solms 1883) es una planta herbácea, perenne, acuática flotante no enraizada, comúnmente encontrada en zonas de clima tropical, donde ha llegado a ser considerada como planta invasiva; prefiere aguas ricas en nutrientes, un $\mathrm{pH}$ de 6 a 8 unidades, y una temperatura de 25 a $27.5^{\circ} \mathrm{C}$ (aunque es capaz de crecer entre 1 y $40{ }^{\circ} \mathrm{C}$ ); no obstante, se presume que presenta sensibilidad al frío (Poma \& Valderrama, 2014). Los sistemas con especies flotantes, como el jacinto de agua, son estanques de profundidad variable ( 0.4 a 1.5 $\mathrm{m}$ ) en los que las macrófitas (en un monocultivo o policultivo) se desarrollan de forma natural (Martelo \& Lara, 2012), removiendo el material orgánico (DBO) y fósforo, oxidando el amonio y reduciendo nitratos, por medio de mecanismos complejos que implican filtración, oxidación bacteriana, sedimentación y precipitación química (Cooper et al., 1996, citados por Delgadillo et al., 2010). De esta forma, el agua residual es depurada lenta y progresivamente durante el periodo de retención (Delgadillo et al., 2010).

Finalmente, la cloración trata de la eliminación selectiva de aquellos organismos que causan enfermedades (MINAM, 2009) y su acción es mejor cuando se parte de agua baja en materia orgánica y sólidos suspendidos (Hernández, 2014). El cloro puede ser aplicado, entre otras formas, en soluciones de hipoclorito de calcio - $\mathrm{Ca}(\mathrm{OCl})_{2}$. Este último es un material granular seco producido a partir de la reacción de la cal y el cloro (AWWA, 1991). Su contenido disponible de cloro activo es de 30 a $70 \%$, y es utilizado como agente blanqueador y desinfectante al igual que otros compuestos oxigenados de cloro (López et al., 2005). El cloro, suministrado en sus diferentes formas, es el desinfectante más utilizado en el tratamiento de agua residual doméstica debido a su poder de inactivación sobre los organismos, a través de la oxidación del material celular; siendo considerada una tecnología bien establecida y eficiente en términos de costo (EPA, 1999).

\section{Materiales y métodos}

Diseño de la investigación

Para el presente estudio se utilizó el siguiente diseño experimental:

Tratamiento preliminar: El afluente pasó a través de una cámara de rejas, cuya finalidad fue retener los sólidos gruesos; siguiendo hacia un proceso de presedimentación en un tanque de almacenamiento de 200 1, el cual permitió la retención de los sólidos suspendidos de menor tamaño, fáciles de decantar (material fino y arena).

Previo al proceso de pre-sedimentación, se realizó el análisis físico, químico y microbiológico del afluente, considerando los siguientes parámetros:

- Análisis físico: temperatura y sólidos totales en suspensión (STS).

- Análisis químico: pH y demanda bioquímica de oxígeno $\left(\mathrm{DBO}_{5}\right)$.

- Análisis microbiológico: coliformes termotolerantes.

Tratamiento con Eisenia foetida (lombrices): A la salida del proceso de pre-sedimentación, el agua residual fue sometida a un tratamiento con E. foetida. El agua residual pasó de forma paralela a dos lombrifiltros de $0.15 \mathrm{~m}^{2}$ de superficie $(50 \mathrm{~cm}$ de largo por $30 \mathrm{~cm}$ de ancho), diseñados con base en la metodología citada por Ramón et al. (2015) y conformados por un lecho de $2 \mathrm{~cm}$ de espesor, donde cohabitaron microorganismos y E. foetida sobre un soporte constituido por tres capas: $25 \mathrm{~cm}$ de aserrín y viruta, $15 \mathrm{~cm}$ de grava, y $10 \mathrm{~cm}$ de bolones, 
añadiéndose una capa adicional de $10 \mathrm{~cm}$ de arena gruesa entre la capa de aserrín y viruta, y la capa de grava, según se muestra en la Fórmula 1. Asimismo, se instaló un sistema de riego por goteo que distribuyó el agua residual en la superficie del lombrifiltro (Figura 1).

Este tratamiento fue evaluado en función de dos tasas de riego diferentes $\left(\mathrm{A}_{1}: 0.5\left[\mathrm{~m}^{3} / \mathrm{dí} a\right] / \mathrm{m}^{2}\right.$, a un caudal de $0.051 / \mathrm{min}$, y $\mathrm{A}_{2:} 1\left[\mathrm{~m}^{3} /\right.$ día $] / \mathrm{m}^{2}$, a un caudal de 0.10 1/min), basadas en los estudios de Ramón et al. (2015), que mostró buenos índices de eficiencia a 0.05 1/min, y Arango (2003), que afirma que se obtienen altos porcentajes de reducción al tratar $1 \mathrm{~m}^{3}$ de agua residual cruda en $1 \mathrm{~m}^{2}$ de biofiltro por día, de donde se puede definir la Fórmula 1. Adicionalmente, se tuvo un grupo control que no fue sometido a tratamiento $\left(\mathrm{A}_{0}\right)$.

A la salida de este primer sistema, al cual se denominó sistema A, se analizaron los efluentes a los 7 días, considerando los mismos parámetros analizados en el afluente.

$$
\mathrm{T}_{\text {riego }}=\mathrm{Q} / \mathrm{A}
$$

\section{Fórmula 1.}

Donde:

$\mathrm{T}_{\text {riego: Tasa de riego; }}$

Q: Caudal de diseño;

A: Área requerida para el tratamiento.

Tratamiento con Eichhornia crassipes (jacinto de agua): A la salida del proceso de tratamiento con E. foetida, el agua residual ingresó de forma paralela a dos estructuras tipo estanque que contenían ejemplares de E. crassipes. Los estanques tenían dimensiones de $90 \mathrm{~cm}$ de largo por $40 \mathrm{~cm}$ de ancho y $40 \mathrm{~cm}$ de profundidad (de acuerdo a la longitud de la raíz de la planta) y fueron diseñados tomando como base a los sistemas propuestos por Vizcaíno \& Fuentes (2016). Cada una de las estructuras fue alimentada por única vez al inicio del ensayo.

Este tratamiento fue evaluado en función a dos tiempos de retención ( $\mathrm{B}_{1}: 7$ días y $\mathrm{B}_{2}$ : 14 días), teniendo un grupo control sin tratamiento $\left(\mathrm{B}_{0}\right)$, efectuándose el análisis de los efluentes considerando los parámetros físicos, químicos y microbiológicos señalados líneas arriba. A la sumatoria del tratamiento con E. foetida + E. crassipes se le denominó sistema AB.

Tratamiento con hipoclorito de calcio: A la salida de la fase de tratamiento con E. crassipes, el agua residual fue sometida a cloración, utilizando hipoclorito de calcio: $\mathrm{Ca}(\mathrm{ClO})_{2}$. Para ello, se utilizaron vasos de precipitados de 11 , a los que se adicionó una dosis de cloro (como cloro activo) de $3.68 \mathrm{mg} / \mathrm{l}\left(\mathrm{C}_{1}\right)$, obtenida mediante la suma de la demanda inicial de cloro $(0.7 \mathrm{mg} / \mathrm{l})$ y el cloro residual, este último calculado según la Fórmula 2 (White, 1985; citada por Metcalf \& Eddy, 1995).

$$
\mathrm{N}_{\mathrm{t}} / \mathrm{N}_{0}=\left(1+0.23 \times \mathrm{C}_{\mathrm{t}} \times \mathrm{t}\right)^{-3}
$$

Fórmula 2.

\section{Donde:}

$\mathrm{N}_{\mathrm{t}}$ : Número de organismos coliformes en el instante t. Considerándose el valor final deseado de $10^{0}$ coliformes fecales/100 $\mathrm{ml}$ de agua residual, considerado como indicativo de que todos los patógenos han muerto. Esto con el fin de dar un margen de seguridad al límite de $<1000$ coliformes fecales/100 $\mathrm{ml}$, establecido en las Directrices sobre la calidad microbiológica de las aguas residuales empleadas en agricultura (OMS, 1989).

$\mathrm{N}_{0}$ : Número de organismos coliformes en el instante 0 . Considerándose un valor de $10^{4}$ coliformes fecales $/ 100 \mathrm{ml}$ de agua residual, obtenidos en base a la concentración inicial de $10^{7} \mathrm{NMP} / 100 \mathrm{ml}$ en el afluente, menos una reducción promedio de 3 escalas logarítmicas de coliformes fecales obtenida en el efluente proveniente de la primera fase del tratamiento con E. foetida.

$\mathrm{C}_{\mathrm{t}}$ : Cloro residual en el instante $\mathrm{t}(\mathrm{mg} / \mathrm{l})$.

t: Tiempo de permanencia: 30 minutos.

Después, se aplicó un mezclado rápido y se dejó reposar durante un periodo de reacción de 30 minutos, de acuerdo a Metcalf \& Eddy (1995). Asi también, se tuvo un grupo control que no fue sometido a tratamiento $\left(\mathrm{C}_{0}\right)$. A la salida de esta fase, se realizó nuevamente el análisis del efluente considerando los parámetros ya mencionados. Al conjunto de tratamientos conformados por E. foetida + E. crassipes + hipoclorito de calcio se le denominó sistema ABC.

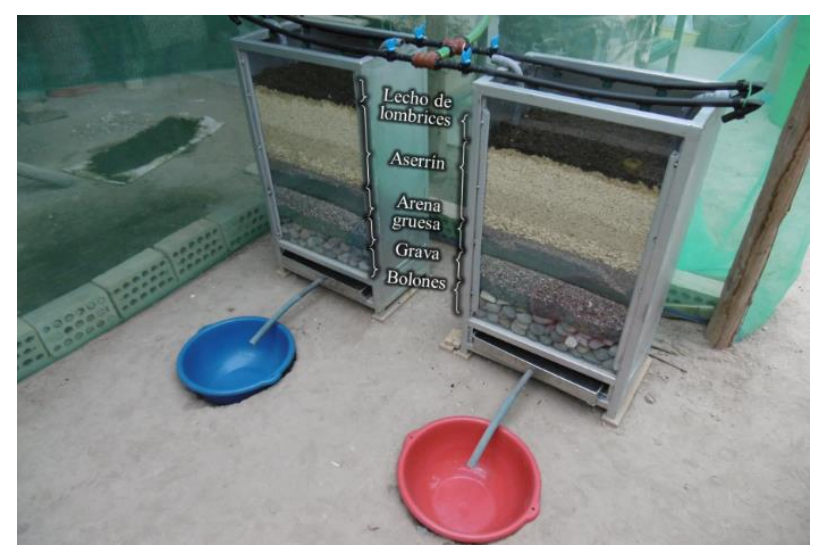

Figura 1. Diseño de los lombrifiltros.

\section{Población y muestra}

La población estuvo conformada por las aguas residuales generadas en el distrito de Moquegua, provincia Mariscal Nieto, región Moquegua. La muestra fue extraída de las Lagunas de estabilización San Antonio (19K 292631.05 E - 8095811.73 S, 1325 
msnm), en horas de máxima y mínima producción para su posterior homogeneización.

Técnicas e instrumentos de recolección de datos

Se aplicó el monitoreo de aguas residuales (afluente y efluentes a la salida de cada uno de los sistemas propuestos). Para ello, se utilizaron como instrumentos de recolección la Ficha de registro de datos de campo y la Ficha de registro de resultados del monitoreo, elaboradas en base a la Resolución Ministerial No 273 2013-VIVIENDA (VIVIENDA, 2013).

Análisis de muestras

Los parámetros físicos, químicos y microbiológicos evaluados fueron seleccionados considerando el Decreto Supremo $\mathrm{N}^{\circ}$ 003-2010-MINAM (MINAM, 2010), y las Directrices sobre la calidad microbiológica de las aguas residuales empleadas en agricultura, recomendadas por la OMS (OMS, 1989).

La medición de parámetros de campo (temperatura y $\mathrm{pH}$ ), la toma y preservación de muestras se realizó considerando las recomendaciones dadas en la Resolución Ministerial $\mathrm{N}^{\mathrm{o}}$ 273-2013-VIVIENDA (VIVIENDA, 2013).

$\mathrm{El}$ análisis de $\mathrm{STS}$ y $\mathrm{DBO}_{5}$ se realizó siguiendo los métodos estándar para el análisis de agua y aguas residuales de la APHA-AWWA-WEF (APHA et al., 2012), utilizando un volumen de $1000 \mathrm{ml}$ por muestra, mientras que el análisis de coliformes termotolerantes se efectuó de acuerdo al método estandarizado de Fermentación de Tubos Múltiples (APHA et al., 2005), el mismo que requiere $250 \mathrm{ml}$ de agua residual por muestra.

Por lo tanto, además de la medición de los parámetros de campo, se tomaron 1) tres muestras en el afluente: una muestra por cada parámetro de análisis en laboratorio, 2) seis muestras a la salida del sistema A: por única vez a los 7 días, 3) 12 muestras a la salida del sistema AB: seis luego de un tiempo de retención de 7 días y seis luego de 14 días, y 4) seis muestras en el sistema ABC: por única vez, luego de un tiempo de reacción de 30 minutos. Así mismo, se trabajó con dos repeticiones por cada parámetro analizado, teniendo un total de 54 muestras.

Procesamiento y análisis de datos

El método estadístico aplicado fue el análisis de varianza (ANOVA), con dos repeticiones para cada indicador de la variable dependiente; y su respectiva prueba de medias de Tukey, a un nivel de significancia del 5\%; haciéndose uso del programa estadístico SPSS.

\section{Resultados}

Características físicas, químicas y microbiológicas del afluente

Los resultados del análisis de los principales parámetros físicos, químicos y microbiológicos del afluente se presentan en la Tabla 1.

Tabla 1. Resultados de la caracterización física, química y microbiológica del afluente.

\begin{tabular}{|c|c|c|}
\hline Parámetro & Unidad & $\begin{array}{c}\text { Valor } \\
\text { obtenido }\end{array}$ \\
\hline \multicolumn{3}{|l|}{ Parámetros físicos } \\
\hline Temperatura & ${ }^{\circ} \mathrm{C}$ & 25.50 \\
\hline $\begin{array}{l}\text { Sólidos totales en } \\
\text { suspensión (STS) }\end{array}$ & $\mathrm{mg} / \mathrm{l}$ & 99.00 \\
\hline \multicolumn{3}{|l|}{ Parámetros químicos } \\
\hline $\mathrm{pH}$ & unidad & 7.38 \\
\hline $\begin{array}{l}\text { Demanda bioquímica de } \\
\text { oxígeno }\left(\mathrm{DBO}_{5}\right)\end{array}$ & $\mathrm{mg} / \mathrm{l}$ & 188.13 \\
\hline $\begin{array}{l}\text { Parámetros } \\
\text { microbiológicos }\end{array}$ & & \\
\hline $\begin{array}{l}\text { Coliformes } \\
\text { termotolerantes }\end{array}$ & $\mathrm{NMP} / 100 \mathrm{ml}$ & $2.40 \times 10^{7}$ \\
\hline
\end{tabular}

Fuente: Laboratorio de Investigación y Servicios (LABINVSERV, 2018); Laboratorio de salud ambiental de la Subgerencia de Salud Ambiental Moquegua (2018).

Características físicas, químicas y microbiológicas de los efluentes a la salida de cada sistema

Los resultados obtenidos del ANOVA, aplicado a los parámetros físicos (temperatura y STS), químicos $\left(\begin{array}{lllll}\mathrm{pH} & \mathrm{y} & \mathrm{DBO}_{5}\end{array}\right)$ y microbiológicos (coliformes termotolerantes) a la salida de cada uno de los sistemas de tratamiento, permitieron observar que la diferencia de medias de éstos fue altamente significativa, según se muestra en la Tabla 2, Tabla 4, y Tabla 6. Por lo tanto, con un nivel de confianza del $99 \%$, al menos uno de los efectos de los sistemas sobre los parámetros de estudio fue distinto en todos los casos, procediéndose a realizar la prueba de Tukey al $5 \%$.

ANÁLISIS FÍsICO

Los resultados de la prueba de Tukey, mostrados en la Tabla 3, expresan que tanto el sistema AB como el sistema $\mathrm{ABC}$ fueron los más efectivos en la reducción del parámetro temperatura, con promedios de 20.50 y $21.90{ }^{\circ} \mathrm{C}$, respectivamente; seguidos en segundo y último lugar por el sistema $\mathrm{A}$ y el agua residual sin tratamiento, lo cual se puede ver reflejado en la Figura 2.

Respecto a los STS, los resultados de la prueba de Tukey, visibles en la Tabla 3 , revelan que tanto el

Tabla 2. ANOVA para los indicadores temperatura y STS, entre sistemas de tratamiento.

\begin{tabular}{|c|c|c|c|c|c|c|c|c|c|}
\hline \multirow{2}{*}{$\begin{array}{c}\text { Fuentes de } \\
\text { variación }\end{array}$} & \multirow{2}{*}{ GL } & \multicolumn{4}{|c|}{ Temperatura } & \multicolumn{4}{|c|}{ STS } \\
\hline & & $\mathrm{SC}$ & $\mathrm{CM}$ & $\mathrm{F}_{\text {cal. }}$ & Sig & $\mathrm{SC}$ & $\mathrm{CM}$ & $\mathrm{F}_{\text {cal. }}$ & Sig \\
\hline Sistemas & 3 & 69.60 & 23.20 & 47.854 & $0.000 * *$ & 16002.23 & 5334.08 & 492.507 & 0.000 ** \\
\hline E. experimental & 14 & 6.79 & 0.49 & & & 151.63 & 10.83 & & \\
\hline Total & 17 & 76.39 & & & & 16153.85 & & & \\
\hline
\end{tabular}


Tabla 3. Prueba de Tukey (0.05) para los indicadores temperatura y STS, entre sistemas de tratamiento.

\begin{tabular}{|c|c|c|c|c|c|c|c|c|}
\hline \multirow[t]{2}{*}{$\mathbf{N}^{\mathbf{o}}$} & \multirow[t]{2}{*}{ Sistema } & \multicolumn{3}{|c|}{ Temperatura } & \multirow[t]{2}{*}{ Sistemas } & \multicolumn{3}{|c|}{ STS } \\
\hline & & $\overline{\mathrm{X}}\left({ }^{\circ} \mathrm{C}\right)$ & Sig & Mérito & & $\overline{\mathrm{X}}(\mathrm{mg} / \mathrm{l})$ & Sig & Mérito \\
\hline 1 & $\mathrm{AB}$ & 20.50 & $\mathrm{a}$ & $1^{\circ}$ & $\mathrm{AB}$ & 5.46 & $\bar{a}$ & $1^{\circ}$ \\
\hline 2 & $\mathrm{ABC}$ & 21.90 & $\mathrm{a}$ & $1^{\mathrm{o}}$ & $\mathrm{ABC}$ & 6.93 & $\mathrm{a}$ & $1^{\mathrm{o}}$ \\
\hline 3 & $\mathrm{~A}$ & 24.68 & $\mathrm{~b}$ & $2^{o}$ & A & 38.50 & $\mathrm{~b}$ & $2^{\circ}$ \\
\hline 4 & Sin tratamiento & 25.50 & $\mathrm{~b}$ & $2^{o}$ & Sin tratamiento & 99.00 & c & $3^{\circ}$ \\
\hline
\end{tabular}

sistema $\mathrm{AB}$ como el sistema $\mathrm{ABC}$ fueron los más efectivos en la remoción de este parámetro, con promedios de 5.46 y $6.93 \mathrm{mg} / \mathrm{l}$, respectivamente; seguidos por el sistema $\mathrm{A}$, y en tercer y último lugar por el agua residual sin tratamiento, lo cual se puede ver reflejado en la Figura 3.

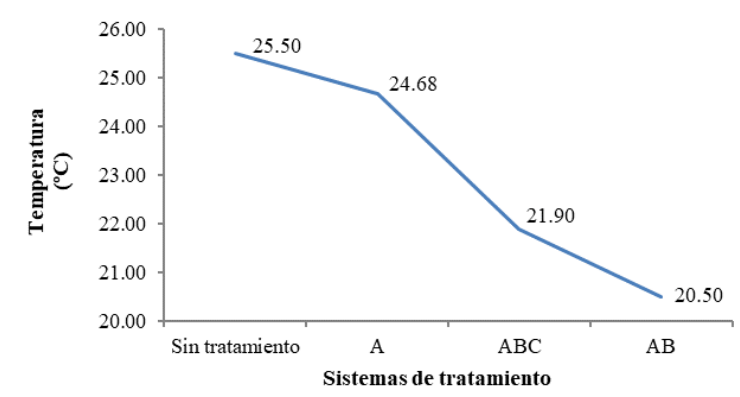

$\mathrm{A}=$ E. foetida $\mathrm{ABC}=$ E. foetida + E. crassipes + hipoclorito de calcio $; \mathrm{AB}=$ E. foetida + E. crassipes.

Figura 2. Prueba de Tukey (0.05) del indicador temperatura, entre sistemas de tratamiento.

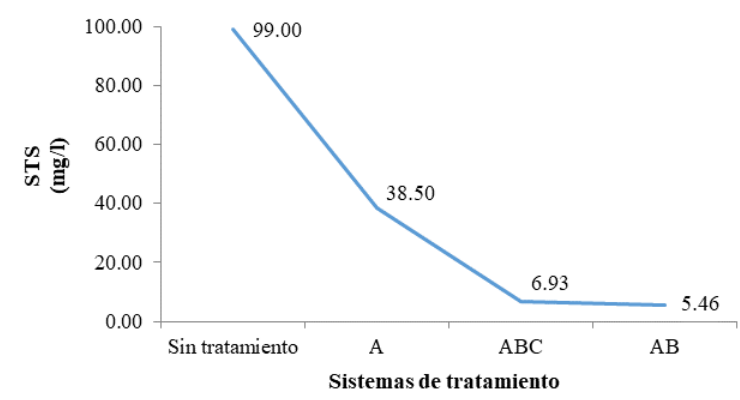

$\mathrm{A}=E$. foetida $; \mathrm{ABC}=E$. foetida + E. crassipes + hipoclorito de calcio; $\mathrm{AB}=$ E. foetida + E. crassipes.

Figura 3. Prueba de Tukey (0.05) para el indicador STS, entre sistemas de tratamiento.

\section{ANÁLISIS QUÍMICO}

Los resultados de la prueba de Tukey, expuestos en la Tabla 5, muestran que tanto el agua residual sin tratamiento como el sistema $\mathrm{AB}$ presentaron los valores de $\mathrm{pH}$ más cercanos a la neutralidad, con promedios de 7.38 y 7.51 unidades, respectivamente; seguidos por el sistema ABC, y en tercer y último lugar por el sistema A, lo cual se puede ver reflejado en la Figura 4.

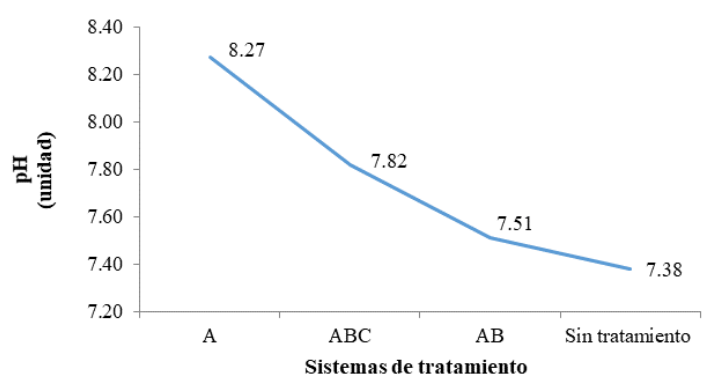

$\mathrm{A}=$ E. foetida $\mathrm{ABC}=$ E. foetida + E. crassipes + hipoclorito de calcio $; \mathrm{AB}=E$. foetida + E. crassipes.

Figura 4. Prueba de Tukey (0.05) para el indicador $\mathrm{pH}$, entre sistemas de tratamiento.

En relación a la $\mathrm{DBO}_{5}$, los resultados de la prueba de Tukey, mostrados en la Tabla 5, expresan que tanto el sistema $\mathrm{ABC}$ como el sistema $\mathrm{AB}$ fueron los más efectivos en la remoción de este parámetro, con promedios de 1.93 y $3.00 \mathrm{mg} / \mathrm{l}$, respectivamente; seguidos por el sistema $\mathrm{A}$, y en tercer y último lugar por el agua residual sin tratamiento, lo cual se puede ver reflejado en la Figura 5.

\section{ANÁLISIS MICROBIOLÓGICO}

Los resultados de la prueba de Tukey, visibles en la Tabla 7, revelan que tanto el sistema $\mathrm{ABC}$ como el sistema $\mathrm{AB}$ fueron los más efectivos en la remoción de coliformes termotolerantes, con promedios de 1.85 y $37.43 \mathrm{NMP} / 100 \mathrm{ml}$; seguidos por el sistema A, y en tercer y último lugar por el agua residual sin

Tabla 4. ANOVA para los indicadores $\mathrm{pH}_{\text {y }} \mathrm{DBO}_{5}$, entre sistemas de tratamiento.

\begin{tabular}{|c|c|c|c|c|c|c|c|c|c|}
\hline \multirow{2}{*}{$\begin{array}{l}\text { Fuentes } \\
\text { variación }\end{array}$} & \multirow{2}{*}{$\begin{array}{l}\mathbf{G} \\
\mathbf{L}\end{array}$} & \multicolumn{4}{|c|}{ pH } & \multicolumn{4}{|c|}{ DBO5 } \\
\hline & & $\mathrm{SC}$ & CM & $\mathrm{F}_{\text {cal. }}$ & Sig & SC & $\mathrm{CM}$ & $\mathrm{F}_{\mathrm{cal}}$ & Sig \\
\hline Sistemas & 3 & 1.82 & 0.61 & 57.076 & $0.000 * *$ & 71991.96 & 23997.32 & 12285.391 & $0.000 * *$ \\
\hline E. experimental & 14 & 0.15 & 0.01 & & & 27.35 & 1.95 & & \\
\hline Total & 17 & 1.97 & & & & 72019.30 & & & \\
\hline
\end{tabular}


Tabla 5. Prueba de Tukey (0.05) para los indicadores $\mathrm{pH}$ y $\mathrm{DBO}_{5}$, entre sistemas de tratamiento.

\begin{tabular}{|c|c|c|c|c|c|c|c|c|}
\hline \multirow[t]{2}{*}{$\mathbf{N}^{\mathbf{o}}$} & \multirow[t]{2}{*}{ Sistema } & \multicolumn{3}{|c|}{ pH } & \multirow[t]{2}{*}{ Sistemas } & \multicolumn{3}{|c|}{$\mathrm{DBO}_{5}$} \\
\hline & & $\overline{\mathrm{X}}$ (unidad) & Sig & Mérito & & $\overline{\mathrm{X}}(\mathrm{mg} / \mathrm{l})$ & Sig & Mérito \\
\hline 1 & Sin tratamiento & 7.38 & $\mathrm{a}$ & $1^{\mathrm{o}}$ & $\mathrm{ABC}$ & 1.93 & $\mathrm{a}$ & $1^{\circ}$ \\
\hline 2 & $\mathrm{AB}$ & 7.51 & $\mathrm{a}$ & $1^{\mathrm{o}}$ & $\mathrm{AB}$ & 3.00 & $\mathrm{a}$ & $1^{\mathrm{o}}$ \\
\hline 3 & $\mathrm{ABC}$ & 7.82 & $\mathrm{~b}$ & $2^{o}$ & A & 93.80 & $\mathrm{~b}$ & $2^{\circ}$ \\
\hline 4 & $\mathrm{~A}$ & 7.38 & a & $1^{\mathrm{o}}$ & $\begin{array}{l}\text { Sin } \\
\text { tratamiento }\end{array}$ & 188.13 & $\mathrm{c}$ & $3^{\circ}$ \\
\hline
\end{tabular}

$\mathrm{A}=$ E. foetida $; \mathrm{AB}=E$. foetida + E. crassipes $; \mathrm{ABC}=E$. foetida + E. crassipes + hipoclorito de calcio

tratamiento, lo cual se puede ver reflejado en la Figura 6.

Grado de reducción de cada parámetro de evaluación por sistema de tratamiento

En las Tablas 8 y 9 se muestra el grado de reducción, respecto al afluente, de cada uno de los parámetros evaluados a la salida de cada sistema de tratamiento.

Tal como se observa en la Tabla 8 , los efluentes de los sistemas $\mathrm{AB}$ y $\mathrm{ABC}$, considerados como los más efectivos en la reducción de los parámetros físicos, presentaron un descenso medio de la temperatura de 5.00 y $3.60{ }^{\circ} \mathrm{C}$, así como una remoción media de STS de 94.48 y $93.01 \%$, respectivamente.

Por otro lado, los efluentes del sistema AB, el cual fue el sistema propuesto que presentó valores de $\mathrm{pH}$ más cercanos a la neutralidad, experimentó un incremento medio de 0.13 unidades respecto al afluente.

Finalmente, los efluentes de los sistemas ABC y $\mathrm{AB}$, considerados como los más efectivos en la remoción de la $\mathrm{DBO}_{5}$ y coliformes termotolerantes, experimentaron una reducción media de 98.98 y $98.41 \%$ de $\mathrm{DBO}_{5}$, respectivamente, y una reducción media del $100.00 \%$ de coliformes termotolerantes en ambos casos.

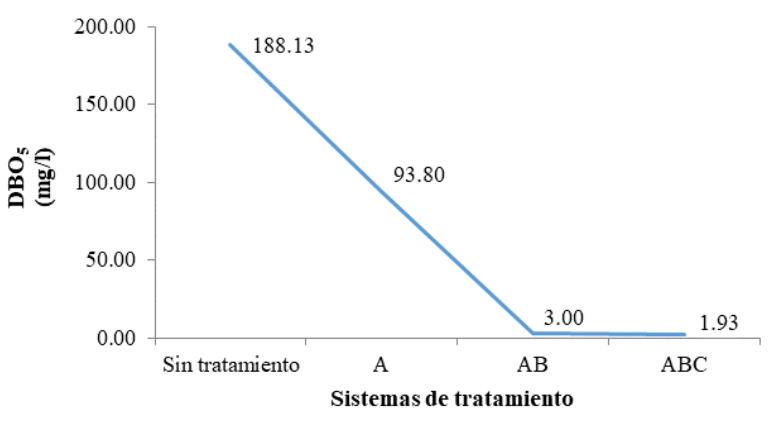

$\mathrm{A}=$ E. foetida $; \mathrm{AB}=E$. foetida + E. crassipes $; \mathrm{ABC}=$ E. foetida + E. crassipes + hipoclorito de calcio.

Figura 5. Prueba de Tukey (0.05) para el indicador $\mathrm{DBO}_{5}$, entre sistemas de tratamiento.
Tabla 6. ANOVA para el indicador coliformes termotolerantes, entre sistemas de tratamiento.

\begin{tabular}{lccccc}
$\begin{array}{c}\text { Fuentes de } \\
\text { variación }\end{array}$ & GL & SC & CM & F $_{\text {cal. }}$ & Sig \\
\hline Sistemas & 2 & $1.02 \times 10^{15}$ & $3.41 \times 10^{14}$ & 936 & $0.000^{* *}$ \\
$\begin{array}{l}\text { E. } \\
\text { experimental }\end{array}$ & 3 & $5.09 \times 10^{9}$ & $3.64 \times 10^{8}$ & & \\
Total & 5 & $1.02 \times 10^{15}$ & & & \\
\hline$* * \mathrm{p}<0.01=$ altamente significativo. & & & \\
\end{tabular}

Tabla 7. Prueba de Tukey (0.05) para el indicador coliformes termotolerantes, entre sistemas de tratamiento.

\begin{tabular}{|c|c|c|c|c|}
\hline $\mathbf{N}^{\mathbf{o}}$ & Sistema & $\begin{array}{c}\overline{\mathbf{X}} \\
(\mathrm{NMP} / 100 \mathrm{ml})\end{array}$ & Sig & Mérito \\
\hline 1 & $\mathrm{ABC}$ & 1.85 & $\mathrm{a}$ & $1^{\circ}$ \\
\hline 2 & $\mathrm{AB}$ & 37.43 & $\mathrm{a}$ & $1^{\mathrm{o}}$ \\
\hline 3 & A & 69500.00 & $\mathrm{~b}$ & $2^{\circ}$ \\
\hline 4 & $\begin{array}{l}\text { Sin } \\
\text { tratamiento }\end{array}$ & 24000000.00 & $\mathrm{c}$ & $3^{\circ}$ \\
\hline
\end{tabular}

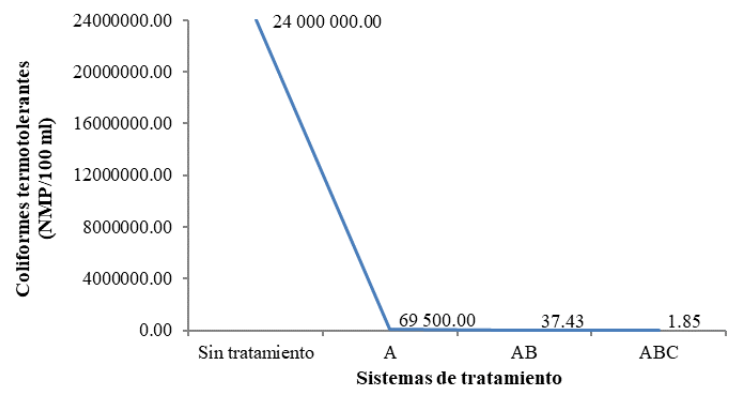

$\mathrm{A}=$ E. foetida $; \mathrm{AB}=$ E. foetida + E. crassipes $; \mathrm{ABC}=$ E. foetida $+E$. crassipes + hipoclorito de calcio.

Figura 6. Prueba de Tukey (0.05) para el indicador coliformes termotolerantes, entre sistemas de tratamiento.

\section{Discusión}

Los resultados de la caracterización física, química y microbiológica del afluente (Tabla 1) mostraron que éste presentaba las características típicas de un agua residual doméstica. Así, presentó una temperatura situada dentro del rango óptimo para el desarrollo de la 
actividad bacteriana (entre 25 y $35^{\circ} \mathrm{C}$ ), además de una concentración de STS considerada como “débil", según Metcalf \& Eddy (2003), citado por Déniz (2010). Adicionalmente presentó un pH neutro, una concentración "media" de $\mathrm{DBO}_{5}$ y una concentración de coliformes termotolerantes, también situada dentro de los valores típicos de un agua residual bruta (Metcalf \& Eddy, 2003; citado por Déniz, 2010).

Los resultados del ANOVA y la prueba de Tukey aplicada entre los tres sistemas de tratamiento propuestos (A: Tratamiento con E. foetida, $\mathrm{AB}$ : Tratamiento con E. foetida + E. crassipes y ABC: Tratamiento con E. foetida $+E$. crassipes + hipoclorito de calcio), y el afluente sin tratamiento, mostraron que existen diferencias significativas entre ellos, arrojando mejores resultados para el sistema $\mathrm{AB}$ en la mayoría de indicadores de la variable dependiente considerados, seguido por ABC. Respecto a este último sistema, cabe destacar que no se han encontrado antecedentes relacionados a la cloración de agua residual con hipoclorito de calcio al nivel propuesto.

En relación al afluente, la temperatura tuvo un descenso de $0.83{ }^{\circ} \mathrm{C}$ para $\mathrm{A}, 5.00{ }^{\circ} \mathrm{C}$ para $\mathrm{AB}$ y $3.60{ }^{\circ} \mathrm{C}$ para $\mathrm{ABC}$, respectivamente, existiendo diferencias estadísticas entre los sistemas propuestos, teniendo $\mathrm{AB}$ y $\mathrm{ABC}$ un mayor efecto en el descenso de los valores de este parámetro.

En cuanto al sistema $\mathrm{AB}$, la temperatura del agua descendió hasta en $4.45^{\circ} \mathrm{C}$ en relación al afluente luego de 7 días de tratamiento; sin embargo, según los estudios realizados por Valderrama et al. (2003) los valores de la temperatura pueden variar a lo largo del día. De forma similar, a los 14 días, la temperatura continuó descendiendo, disminuyendo hasta en $5.90{ }^{\circ} \mathrm{C}$ con relación del afluente.

Los STS alcanzaron una eficiencia de remoción de $61.11 \%$ para el sistema A, $94.48 \%$ para AB y $93.01 \%$ para $\mathrm{ABC}$, considerándose tanto a $\mathrm{AB}$ como a $\mathrm{ABC}$ como los sistemas que tuvieron un mayor efecto en la reducción de este parámetro.

Respecto al sistema AB, los STS fueron removidos hasta una media de $2.95 \mathrm{mg} / \mathrm{l}$ en $\mathrm{A}_{1} \mathrm{~B}_{1}(97.02 \%$ de remoción) y $5.85 \mathrm{mg} / \mathrm{l}$ (94.09\% de remoción) en $\mathrm{A}_{2} \mathrm{~B}_{1}$. Esta alta eficiencia en la remoción de sólidos suspendidos se debería a que las raíces de las macrófitas flotantes actúan como un medio de filtración/adsorción excelente (Martelo \& Lara, 2012). Por el contrario, luego de 14 días de retención, los STS se incrementaron con respecto a la semana anterior. Al respecto, Vizcaíno \& Fuentes (2016) reportan un mayor porcentaje de remoción de este parámetro, alcanzando el $100 \%$ de remoción en un tiempo de experimentación de 45 días; cabe resaltar que inician su estudio con niveles de STS mayores al presente estudio (384 mg/l).

$\mathrm{El} \mathrm{pH}$, por otro lado, mantuvo valores superiores en todos los sistemas, respecto al agua sin tratar, alcanzando medias de $8.27,7.51$ y 7.82 unidades en los afluentes de $\mathrm{A}, \mathrm{AB}$ y $\mathrm{ABC}$, respectivamente. Valores elevados de $\mathrm{pH}$ en el primer sistema fueron relacionados a la actividad de las glándulas calcíferas de E. foetida (encargadas de la segregación y descarga de carbonato cálcico al alimento) (Somarriba \& Guzmán, 2004), descendiendo durante su tratamiento con E. crassipes, e incrementándose nuevamente a la salida del tratamiento con hipoclorito de calcio, debido a las propiedades de "esta sal [que] al disolverse eleva el pH de la solución" (Castro, s/f.).

Por tal motivo, fue el sistema $\mathrm{AB}$ el que presentó valores de $\mathrm{pH}$ más cercanos a la neutralidad. En comparación, Valderrama et al. (2003) obtuvieron valores ligeramente más bajos y cercanos a la neutralidad a los 6 días de retención, empleando solo E. crassipes, con 7.36 unidades, lo cual se debería a que las macrófitas limitan la actividad fotosintética de las microalgas, al restringir el paso de la luz, impidiendo que disminuyan las concentraciones de dióxido de carbono $\left(\mathrm{CO}_{2}\right)$ disueltos en el agua (Valderrama, 1996).

La $\mathrm{DBO}_{5}$ presentó una eficiencia de remoción de $50.14 \%$ para $\mathrm{A}, 98.41 \%$ para $\mathrm{AB}$ y $98.98 \%$ para $\mathrm{ABC}$, demostrando estadísticamente que $\mathrm{AB}$ y $\mathrm{ABC}$ fueron los sistemas más eficientes en la reducción de la concentración de la $\mathrm{DBO}_{5}$ del afluente.

En comparación a la reducción experimentada en el sistema AB, Vizcaíno \& Fuentes (2016) obtuvieron un menor porcentaje de remoción, alcanzando un máximo del $91 \%$. Por otro lado, Martelo \& Lara (2012) afirman que la eficiencia de remoción de la DBO oscila de 37 a 95.1\% utilizando solo E. crassipes. Esta reduccción estaría relacionada con la remoción de materia orgánica por microorganismos asociados a la zona radicular de planta y a la acción de la misma como filtro (Valderrama, 1996).

En lo que corresponde al sistema $\mathrm{ABC}$, la concentración de la $\mathrm{DBO}_{5}$ fue notablemente menor que la del grupo control, encontrándose ya sobre los límites de detección del método de análisis para este parámetro.

De manera similar, las coliformes termotolerantes experimentaron una reducción de $99.71 \%$ en $\mathrm{A}$, y de $100.00 \%$ tanto en $\mathrm{AB}$ como $\mathrm{ABC}$, situación que fue reafirmada estadísticamente a través de la prueba de Tukey, definiéndose que tanto $\mathrm{AB}$ como $\mathrm{ABC}$ fueron los sistemas que tuvieron mayor efecto en la reducción de este parámetro.

Respecto al sistema $\mathrm{AB}$, los resultados obtenidos a los 7 días de retención mostraron que se experimentó una reducción significativa de coliformes termotolerantes, alcanzando el $100.00 \%$ de remoción tanto en $\mathrm{A}_{1} \mathrm{~B}_{1}$ como en $\mathrm{A}_{2} \mathrm{~B}_{1}$. Al respecto, estos valores superaron a los obtenidos por Vizcaíno \& Fuentes (2016), los cuales alcanzaron el $99 \%$ de remoción bajo la denominación de coliformes fecales en 45 días de experimentación. Asimismo, Valderrama et al. (2003) obtuvieron valores similares empleando solo E. crassipes, notándose en este caso la disminución promedio del parámetro coliformes fecales en un 
$99.00 \%$ a los 6 días de retención o estancamiento. La alta eficiencia en la remoción microbiana se debería al oxígeno que las plantas transfieren de la atmósfera hacia su zona radicular generando condiciones aerobias (Martelo \& Lara, 2012).

Así mismo, el número de coliformes termotolerantes, a los 14 días, descendió de forma importante en relación al grupo control y a la semana anterior, siendo removidas prácticamente en su totalidad.

Finalmente, en el sistema $\mathrm{ABC}$ se alcanzaron valores sobre el límite de cuantificación del método analítico aplicado para coliformes termotolerantes, encontrándose prácticamente ausentes en el efluente. Al respecto, Reyes (2016) también reportó una reducción del $99.9999 \%$ de este parámetro luego de un tratamiento terciario de desinfección con cloro (cloro gaseoso) en los meses de primavera y verano.

\section{Conclusiones}

Se evaluaron tres sistemas de depuración, determinándose como el más eficiente para el tratamiento de aguas residuales domésticas al sistema conformado por lombrices (Eisenia foetida) + jacinto de agua (Eichhornia crassipes), con un descenso medio de $5{ }^{\circ} \mathrm{C}, 94.48 \%$ de STS, $98.41 \%$ de $\mathrm{DBO}_{5}, 100.00 \%$ de coliformes termotolerantes, y un $\mathrm{pH}$ final medio de 7.51 .

A través del análisis de los parámetros considerados, se determinaron las características físicas, químicas y microbiológicas del afluente, conociéndose que el agua residual presentó una temperatura de $25.50{ }^{\circ} \mathrm{C}$, un $\mathrm{pH}$ neutro de 7.38 , y concentraciones típicas de $99.00 \mathrm{mg} / \mathrm{l}$ de STS, 188.13 $\mathrm{mg} / \mathrm{l}$ de $\mathrm{DBO}_{5}$, y $2.40 \times 10^{7} \mathrm{NMP} / 100 \mathrm{ml}$ de coliformes termotolerantes.

Se analizaron las características físicas, químicas y microbiológicas de los efluentes, determinándose que el sistema más eficiente fue el integrado por E. foetida + E. crassipes, seguido por el sistema E. foetida + E. crassipes + hipoclorito de calcio y, por último, el sistema con E. foetida.

\section{Agradecimientos}

Nuestro más profundo agradecimiento al Laboratorio de Ingeniería Ambiental de la Universidad José Carlos Mariátegui, por permitir el uso de sus instalaciones, equipos y materiales; y al Dr. Luis Herrera, quien gustosamente compartió sus valiosos conocimientos en el manejo de la lombriz roja californiana, proporcionándonos, además, las especies utilizadas en el presente estudio.

\section{Literatura citada}

AWWA (American Water Works Association). 1991. De regreso a la guía básica para la desinfección con cloro (original en inglés). Recuperado de http://www.bvsde.paho.org/eswww/fulltext/repind55/ba ckbasi/backbasi.html.

APHA (American Public Health Association), AWWA (American Water Works Association) \& WEF (Water Environment Federation). 2005. Standard Methods "Examination of Water and Wastewater". Vigésima primera edición.

APHA (American Public Health Association), AWWA (American Water Works Association) \& WEF (Water Environment Federation). 2012. Standard Methods "Examination of Water and Wastewater". Vigésima segunda edición.

Arango J. 2003. Evaluación ambiental del Sistema Tohá en la remoción de Salmonella en aguas servidas domésticas. Tesis (Magister en Gestión y Planificación Ambiental). Universidad de Chile. Santiago, Chile.

Castro E. s/f. Principios de control microbiológico con oxidantes. Recuperado de https://www.micof.es/bd/archivos/archivo1956.pdf.

Delgadillo O., Camacho A., Pérez L. \& Andrade M. 2010. Depuración de aguas residuales por medio de humedales artificiales. Centro andino para la gestión y uso del agua (Centro AGUA) / Universidad Mayor de San Simón. Cochabamba, Bolivia. https://core.ac.uk/download/pdf/48017573.pdf.

Déniz F. 2010. Análisis estadístico de los parámetros DQO, $\mathrm{DBO}_{5}$ y SS de las aguas residuales urbanas en el ensuciamiento de las membranas de ósmosis inversa. Tesis doctoral. Universidad Las Palmas de Gran Canaria. Las Palmas de Gran Canaria, España.

EPA (United States Environmental Protection Agency). 1999. Folleto Informativo de tecnología de aguas residuales: Desinfección con cloro. EPA 832-F-99-063. Washington D.C., Estados Unidos. https://www.epa.gov/sites/production/files/201506/documents/cs-99-063.pdf.

García M. \& Solano V. 2005. Manual cría de la lombriz de tierra: una alternativa ecológica y rentable. San Pablo, Colombia. https://books.google.com.pe/books?isbn=9588233321.

Hernández J. 2014. Cloración en los procesos de tratamiento de aguas residuales urbanas. http://www.microlabindustrial.com/blog/cloracion-enlos-procesos-de-tratamiento-de-aguas-residuales.

LABINVSERV (Laboratorio de Investigación y Servicios). 2018. Informe de ensayo físico químico. Reportes $\mathrm{N}^{\mathrm{os}}$ 19142-18, 19143-18 y 19144-18. Moquegua, Perú.

Laboratorio de Salud Ambiental de la Subgerencia de Salud Ambiental Moquegua. 2018. Informes de ensayo $\mathrm{N}^{\text {os }}$ 061.18, 065.18, 099.18, 100.18, 109.18 у 130.18 . Moquegua, Perú.

López L., Monier F. \& Labrada B. 2005. Estudio de la obtención electrolítica "in situ" de disoluciones acuosas de hipoclorito de sodio. Tecnología Química, 25(2): 3945. https://www.redalyc.org/pdf/4455/445543747006.pdf.

Martelo J. \& Lara J. 2012. Macrófitas flotantes en el tratamiento de aguas residuales; una revisión del estado del arte. Ingeniería y Ciencia, 8(15): 221-243. http://www.redalyc.org/pdf/835/83524069011.pdf. 
Metcalf \& Eddy. 1995. Ingeniería de aguas residuales: tratamiento, vertido y reutilización. Interamericana de España, S.A.U. España. (Juan de Dios Trujillo, trad. Obra original publicada en 1991).

MINAGRI (Ministerio de Agricultura, PE). 2010. Decreto Supremo No 001-2010-AG: Reglamento de la Ley de Recursos Hídricos (Ley $N^{\circ}$ 29338). Diario Oficial El Peruano, 24 de marzo, 27(10937): 416022-416052. Recuperado https://elperuano.pe/NormasElperuano/2010/03/24/4725 61-3.html.

MINAM (Ministerio del Ambiente, PE). 2009. Manual para municipios ecoeficientes. ENOTRIA S.A. Lima, Perú. http://www.minam.gob.pe/calidadambiental/wpcontent/uploads/sites/22/2013/10/manual_para_municipi os_ecoeficientes.pdf.

MINAM (Ministerio del Ambiente, PE). 2010. Decreto Supremo $\mathrm{N}^{\circ}$ 003-2010-MINAM: Aprueba Límites Máximos Permisibles para los efluentes de Plantas de Tratamiento de Aguas Residuales Domésticas o Municipales. Diario Oficial El Peruano, 17 de marzo, 27(10930): 415675-415676. Recuperado de https://elperuano.pe/NormasElperuano/2010/03/17/4694 46-2.html.

OEFA (Organismo de Evaluación y Fiscalización Ambiental, PE). 2014. Fiscalización ambiental en aguas residuales. https://www.oefa.gob.pe/?wpfb_dl=7827. Lima, Perú.

OMS (Organización Mundial de la Salud). 1989. Directrices sanitarias sobre el uso de aguas residuales en agricultura y acuicultura: informe de un Grupo Científico de la OMS (Serie de Informes Técnicos $\mathrm{N}^{\mathbf{0}}$ 778). Organización Mundial de la Salud. Ginebra, Suiza. (Organización Panamericana de la Salud, trad. Obra original publicada en 1989). https://apps.who.int/iris/handle/10665/39333.

Poma V. \& Valderrama A. 2014. Estudio de los parámetros fisicoquímicos para la fitorremediación de cadmio (II) y mercurio (II) con la especie Eichhornia crassipes (jacinto de agua). Revista de la Sociedad Química del Perú, 80(3): 164-173. http://www.scielo.org.pe/scielo.php?pid=S1810634X2014000300003\&script=sci_arttext.

Ramón J., León J. \& Castillo N. 2015. Diseño de un sistema alternativo para el tratamiento de aguas residuales urbanas por medio de la técnica de lombrifiltros utilizando la especie Eisenia foetida. Revista Mutis, 5(1): 46-54. https://core.ac.uk/download/pdf/194855493.pdf.
Reyes M. 2016. Uso del cloro en las plantas de tratamiento de aguas residuales domesticas: desinfección y formación de subproductos. Tesis (Maestro en Ciencias en Gestión Ambiental). Instituto Politécnico Nacional. Durango, México.

Rodríguez F. 2005. Lombricultura para pequeños emprendedores: manual teórico práctico para el manejo comercial de la lombriz roja californiana. Editorial La Quimera, Argentina. http://books.google.com/books?id=F00Ky06O2qwC.

Somarriba R. \& Guzmán F. 2004. Guía de lombricultura. Universidad Nacional Agraria. Managua, Nicaragua. http://repositorio.una.edu.ni/2409/1/nf04s693.pdf.

UOLASAT (Unidad Operativa Laboratorio Ambiental San Agustín de Torata). 2018. Informe de ensayo. Moquegua, Perú. Informes $\mathrm{N}^{\text {os }}$ A-002/2018, A-004/2018 y A006/2018.

Valderrama L., Campos C., Velandia S. \& Zapata N. 2003. Evaluación del efecto del tratamiento con plantas acuáticas (E. crassipes, Lemma sp. y L. laevigatum) en la remoción de indicadores de contaminación fecal en aguas residuales domésticas. Seminario Internacional sobre Métodos Naturales para el Tratamiento de Aguas Residuales.

Valderrama T. 1996. Uso de dos especies de macrófitas acuáticas, Limnobium laevigatum y Eichhornia crassipes para el tratamiento de aguas residuales agro industriales. Universitas Scientiarum, 3(1-2): 83-97. http://revistas.javeriana.edu.co/index.php/scientarium/art icle/view/5058.

VIVIENDA (Ministerio de Vivienda, Construcción y Saneamiento, PE). 2013. Resolución Ministerial No 2732013-VIVIENDA: Aprueban el Protocolo de Monitoreo de la Calidad de los Efluentes de las Plantas de Tratamiento de Aguas Residuales Domésticas o Municipales - PTAR. Diario Oficial El Peruano, 30 de octubre, 30(12636): 506024-506025. Recuperado de https://elperuano.pe/NormasElperuano/2013/10/30/1007 030-1.html.

Vizcaíno L. \& Fuentes N. 2016. Efectos de Eisenia foetida y Eichhornia crassipes en la remoción de materia orgánica, nutrientes y coliformes en efluentes domésticos. Rev. U.D.C.A. Act. \& Div. Cient., 19(1): 189198. https://revistas.udca.edu.co/index.php/ruadc/article/ view/225/1324.

http://www.scielo.org.co/pdf/rudca/v19n1/v19n1a22.pdf. 
Enero - Julio 2021

DEPURACIÓN DE AGUAS RESIDUALES DOMÉSTICAS EN MOQUEGUA / PERÚ

Tabla 8. Grado de reducción de la temperatura, STS y pH a la salida de cada sistema de tratamiento.

\begin{tabular}{|c|c|c|c|c|c|c|c|}
\hline \multirow[b]{2}{*}{ Sistema } & \multirow[b]{2}{*}{ Tratamiento } & \multicolumn{2}{|c|}{ Temperatura } & \multicolumn{2}{|c|}{ STS } & \multirow[b]{2}{*}{$\begin{array}{c}\bar{X} \\
(\mathrm{ud})\end{array}$} & \multirow{2}{*}{$\begin{array}{l}\text { pH } \\
\text { Incremento } \\
\text { medio }^{c} \\
\text { (unidad) }\end{array}$} \\
\hline & & $\overline{\mathrm{X}}\left({ }^{\circ} \mathrm{C}\right)$ & $\begin{array}{c}\text { Descenso } \\
\text { medio }^{\mathrm{a}} \\
\left({ }^{\circ} \mathrm{C}\right)\end{array}$ & $\begin{array}{c}\overline{\mathrm{X}} \\
(\mathrm{mg} / \mathrm{l})\end{array}$ & $\begin{array}{c}\text { Remoción } \\
\text { media }^{b} \\
(\%)\end{array}$ & & \\
\hline A: E. foetida & $\mathrm{A}_{1}: 0.5\left(\mathrm{~m}^{3} / \mathrm{día}\right) / \mathrm{m}^{2}$ & 25.25 & 0.82 & 33.00 & 61.11 & 8.31 & 0.89 \\
\hline & $\mathrm{A}_{2}: 1\left(\mathrm{~m}^{3} /\right.$ día $) / \mathrm{m}^{2}$ & 24.10 & & 44.00 & & 8.23 & \\
\hline \multirow[t]{4}{*}{$\begin{array}{l}\text { AB: E. foetida + } \\
\text { E. crassipes }\end{array}$} & $\begin{array}{l}\mathrm{A}_{1} \mathrm{~B}_{1}: 0.5\left(\mathrm{~m}^{3} / \mathrm{día}\right) / \mathrm{m}^{2}+7 \text { días de } \\
\text { retención }\end{array}$ & 21.05 & 5.00 & 2.95 & 94.48 & 7.72 & 0.13 \\
\hline & $\begin{array}{l}\mathrm{A}_{2} \mathrm{~B}_{1}: 1\left(\mathrm{~m}^{3} / \mathrm{día}\right) / \mathrm{m}^{2}+7 \text { días de } \\
\text { retención }\end{array}$ & 21.35 & & 5.85 & & 7.43 & \\
\hline & $\begin{array}{l}\mathrm{A}_{1} \mathrm{~B}_{2}: 0.5\left(\mathrm{~m}^{3} / \mathrm{día}\right) / \mathrm{m}^{2}+14 \text { días de } \\
\text { retención }\end{array}$ & 19.60 & & 6.85 & & 7.50 & \\
\hline & $\begin{array}{l}\mathrm{A}_{2} \mathrm{~B}_{2}: 1\left(\mathrm{~m}^{3} / \text { día }\right) / \mathrm{m}^{2}+14 \text { días de } \\
\text { retención }\end{array}$ & 20.00 & & 6.20 & & 7.41 & \\
\hline $\begin{array}{l}\text { ABC: E. foetida }+ \\
\text { E. crassipes }+\end{array}$ & $\begin{array}{l}\mathrm{A}_{1} \mathrm{~B}_{2} \mathrm{C}_{1}: 0.5\left(\mathrm{~m}^{3} / \mathrm{día}\right) / \mathrm{m}^{2}+14 \text { días } \\
\text { de retención }+\mathrm{Ca}(\mathrm{ClO})_{2}\end{array}$ & 21.85 & 3.60 & 7.60 & 93.01 & 7.86 & 0.44 \\
\hline hipoclorito de calcio & $\begin{array}{l}\mathrm{A}_{2} \mathrm{~B}_{2} \mathrm{C}_{1}: 1\left(\mathrm{~m}^{3} / \text { día }\right) / \mathrm{m}^{2}+14 \text { días de } \\
\text { retención }+\mathrm{Ca}(\mathrm{ClO})_{2}\end{array}$ & 21.95 & & 6.25 & & 7.78 & \\
\hline \multicolumn{2}{|l|}{ Sin tratamiento } & 25.50 & 0.00 & 99.00 & 0.00 & 7.38 & 0.00 \\
\hline
\end{tabular}

Tabla 9. Grado de reducción de la $\mathrm{DBO}_{5}$ y coliformes termotolerantes a la salida de cada sistema de tratamiento.

\begin{tabular}{|c|c|c|c|c|c|}
\hline \multirow[b]{2}{*}{ Sistema } & \multirow[b]{2}{*}{ Tratamiento } & \multicolumn{2}{|c|}{$\mathrm{DBO}_{5}$} & \multicolumn{2}{|c|}{ Coliformes termotolerantes } \\
\hline & & $\begin{array}{c}\overline{\mathrm{X}} \\
(\mathrm{mg} / \mathrm{l})\end{array}$ & $\begin{array}{l}\text { Remoción } \\
\text { media }^{\mathrm{a}}(\%)\end{array}$ & $\begin{array}{c}\overline{\mathrm{X}} \\
(\mathrm{NMP} / 100 \mathrm{ml})\end{array}$ & $\begin{array}{l}\text { Remoción } \\
\text { media }^{\mathrm{a}}(\%)\end{array}$ \\
\hline \multirow[t]{2}{*}{ A: E. foetida } & $\mathrm{A}_{1}: 0.5$ (m³/día) $/ \mathrm{m}^{2}$ & 93.38 & 50.14 & 105000.00 & 99.71 \\
\hline & $\mathrm{A}_{2}: 1\left(\mathrm{~m}^{3} /\right.$ día $) / \mathrm{m}^{2}$ & 94.23 & & 34000.00 & \\
\hline \multirow[t]{4}{*}{$\begin{array}{l}\text { AB: E. foetida } \\
\text { E. crassipes }\end{array}$} & $\begin{array}{l}\mathrm{A}_{1} \mathrm{~B}_{1}: 0.5\left(\mathrm{~m}^{3} / \mathrm{día}\right) / \mathrm{m}^{2}+7 \text { días de } \\
\text { retención }\end{array}$ & 3.80 & 98.41 & 125.00 & 100.00 \\
\hline & $\begin{array}{l}\mathrm{A}_{2} \mathrm{~B}_{1}: 1\left(\mathrm{~m}^{3} / \mathrm{dí} a\right) / \mathrm{m}^{2}+7 \text { días de } \\
\text { retención }\end{array}$ & 3.20 & & 21.00 & \\
\hline & $\begin{array}{l}\mathrm{A}_{1} \mathrm{~B}_{2}: 0.5\left(\mathrm{~m}^{3} / \mathrm{dí} a\right) / \mathrm{m}^{2}+14 \text { días de } \\
\text { retención }\end{array}$ & 3.00 & & 1.80 & \\
\hline & $\begin{array}{l}\mathrm{A}_{2} \mathrm{~B}_{2}: 1\left(\mathrm{~m}^{3} / \text { día }\right) / \mathrm{m}^{2}+14 \text { días de } \\
\text { retención }\end{array}$ & 2.00 & & 1.90 & \\
\hline $\begin{array}{l}\mathrm{ABC}: \text { E. foetida }+ \\
\text { E. crassipes }+\end{array}$ & $\begin{array}{l}\mathrm{A}_{1} \mathrm{~B}_{2} \mathrm{C}_{1}: 0.5\left(\mathrm{~m}^{3} / \mathrm{día}\right) / \mathrm{m}^{2}+14 \text { días } \\
\text { de retención }+\mathrm{Ca}(\mathrm{ClO})_{2}\end{array}$ & 2.00 & 98.98 & 1.80 & 100.00 \\
\hline hipoclorito de calcio & $\begin{array}{l}\mathrm{A}_{2} \mathrm{~B}_{2} \mathrm{C}_{1}: 1\left(\mathrm{~m}^{3} / \mathrm{día}\right) / \mathrm{m}^{2}+14 \text { días de } \\
\text { retención }+\mathrm{Ca}(\mathrm{ClO})_{2}\end{array}$ & 1.85 & & 1.90 & \\
\hline Sin tratamiento & & 188.13 & 0.00 & 24000000 & 0.00 \\
\hline
\end{tabular}

${ }^{\mathrm{a}}$ Remoción media respecto al afluente.

Fuente: Adaptado de Laboratorio de Investigación y Servicios (LABINVSERV, 2018); Unidad Operativa Laboratorio Ambiental San Agustín de Torata (UOLASAT, 2018); Laboratorio de salud ambiental de la Subgerencia de Salud Ambiental Moquegua (2018).

\footnotetext{
${ }^{1}$ Escuela Profesional de Ingeniería Ambiental. Facultad de Ingeniería y Arquitectura. Universidad José Carlos Mariátegui. Campus San Antonio s/n, Moquegua-Perú. dkcaceresp86@ gmail.com.

${ }^{2}$ Escuela Profesional de Ingeniería Ambiental. Facultad de Ingeniería y Arquitectura. Universidad José Carlos Mariátegui. Campus San Antonio s/n, Moquegua-Perú. gisel_kindesses@ hotmail.com.

${ }^{3}$ Docente ordinario. Dr. en Medio Ambiente y Desarrollo Sostenible. Director de la Escuela Profesional de Ingeniería Ambiental. Universidad José Carlos Mariátegui. Campus San Antonio s/n, Moquegua-Perú. ebedoya@ujcm.edu.pe.
} 